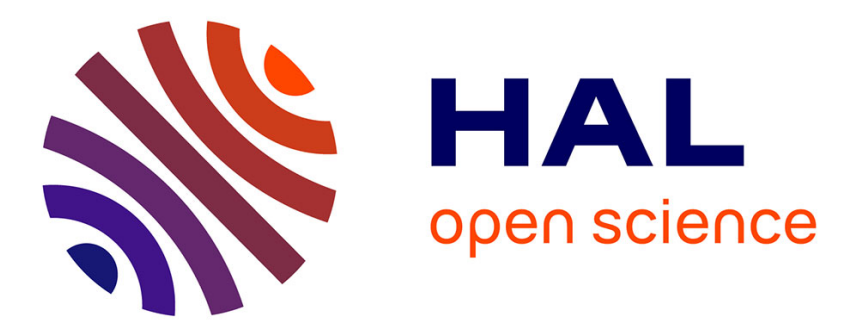

\title{
Jean Radvanyi, Marlène Laruelle, La Russie, entre peurs et défis
}

Victor Violier

\section{To cite this version:}

Victor Violier. Jean Radvanyi, Marlène Laruelle, La Russie, entre peurs et défis. Lectures, 2016, 10.4000/lectures.21371 . hal-03010580

\section{HAL Id: hal-03010580 \\ https://hal.parisnanterre.fr/hal-03010580}

Submitted on 17 Nov 2020

HAL is a multi-disciplinary open access archive for the deposit and dissemination of scientific research documents, whether they are published or not. The documents may come from teaching and research institutions in France or abroad, or from public or private research centers.
L'archive ouverte pluridisciplinaire HAL, est destinée au dépôt et à la diffusion de documents scientifiques de niveau recherche, publiés ou non, émanant des établissements d'enseignement et de recherche français ou étrangers, des laboratoires publics ou privés. 


\section{Marlène Laruelle, Jean Radvanyi, La Russie, entre peurs et défis \\ Victor Violier}

Doctorant et ATER de science politique à l'Université Paris Ouest Nanterre et
membre de l'Institut des Sciences sociales du Politique (UMR 7220-CNRS)

$12 / 09 / 2016$

Avec cet ouvrage rédigé à quatre mains, Marlène Laruelle et Jean Radvanyi nous livrent une synthèse, concise mais néanmoins riche, des mutations politiques, sociales et économiques survenues en Russie depuis la chute de l'Union soviétique. Son intérêt réside notamment dans l'actualisation des derniers développements synthétiques sur la Russie postsoviétique $^{1}$ tout en y intégrant des réflexions sur une actualité riche et complexe (JO de Sotchi, annexion de la Crimée en 2014, crise économique de 2015, etc.). Organisée en sept chapitres thématiques, la réflexion balaie un vaste spectre des transformations qu'à connues la Russie post-soviétique, des difficultés afférentes à la gestion par la fédération de son immense territoire à sa place dans les relations internationales, ses perspectives géopolitiques et grandes orientations stratégiques, en passant par la crise identitaire d'une population déclinante, les inégalités sociales et la fragmentation de la société ainsi que l'évolution du régime politique et la situation économique du pays.

Le premier chapitre de l'ouvrage débute par l'évocation du « syndrome post-impérial » russe et des difficultés que rencontre le pays pour gérer son territoire à l'heure où le pouvoir a rompu pour la seconde fois ${ }^{2}$ avec la promesse d'intangibilité des frontières issues de l'Union soviétique de Boris Eltsine, en annexant la Crimée au mois de mars 2014. Si les différends territoriaux avec ses voisins sont nombreux, la Russie peine à maîtriser sa propre démographie et le défi colossal que lui impose le peuplement de son territoire sans

${ }^{1}$ On peut notamment citer l'ouvrage collectif La Russie contemporaine paru en 2009 aux éditions Karthala, sous la direction de Gilles Favarel-Garrigues et Kathy Rousselet, qui regroupe les contributions d'une quinzaine de chercheurs spécialistes du sujet et constitue un ouvrage de référence, ou, dans un autre registre, plus concis, le repère paru aux éditions La Découverte en 2008 et écrit par Françoise Daucé, La Russie postsoviétique.

${ }^{2}$ La première fois renvoyant au conflit russo-géorgien de 2008 qui s'est soldé par la reconnaissance par la Fédération de Russie, le 26 août 2008, de l'indépendance de l'Ossétie du Sud et de l'Abkhazie. 
commune mesure. Les régions septentrionales et orientales du pays aux conditions climatiques et économiques difficiles sont frappées par un fort déficit démographique, au point d'évoquer, dans le cas de l'extrême orient principalement, jusqu'à un phénomène de désertification. Ce préoccupant constat n'épargne pas même la capitale de la région, Vladivostok, qui a perdu près de 7\% de ses habitants depuis 1991. Ces phénomènes de dépeuplement sont entretenus et renforcés par la faiblesse des réseaux de communication et la terrible disproportion de richesse entre les différentes régions dont Moscou et son oblast représentant à lui seul plus du quart de la richesse nationale - sont le symbole le plus manifeste. Le pouvoir politique en porte sans nul doute une grande part de responsabilité tant l'hypercentralisation politique quasi séculaire a alimenté l'hypertrophie de la capitale. La politique régionale du pouvoir, administrée depuis le centre et dans une démarche hiérarchique typiquement soviétique, n'a quant à elle jamais offert d'alternative à cette modalité de gestion de l'immensité.

Le thème de l'identité fait l'objet du deuxième chapitre qui présente d'abord le débat identitaire ayant (tu répètes deux fois qui sinon, pas très joli) pris place à la chute de l'Union soviétique entre partisans d'une identité civique au sein d'une fédération rassemblant l'ensemble des « russiens » et défenseurs de la création d'un nouvel État-nation « russe ». L'arrivée au pouvoir de Vladimir Poutine bouleverse la donne dès lors que celui-ci développe un nouveau discours politique, résolument patriote, reposant sur la réhabilitation du passé soviétique et le rôle croissant de l'Église orthodoxe, promu « bras droit de l'État » (p. 35). Cette fragilisation du consensus russien est accentuée par l'aura nouvelle des intellectuels et hommes politiques nationalistes qui mettent en garde contre le spectre de la «dé-russification » qui hanterait le pays. Dans une société de plus en plus en proie à la xénophobie, ces discours continuent de mobiliser, comme en témoigne par exemple la forte popularité du juriste et blogueur d'opposition «nationale-démocratique », Alexeï Navalny. Néanmoins, et parallèlement à la «fuite des cerveaux » qui a vu plus de 4 millions de départs depuis 1991 parmi la population la plus jeune et la plus diplômée, la Russie est devenue une terre d'immigration économique, notamment en provenance des anciennes républiques soviétiques. Deux millions de citoyens étrangers travaillent ainsi officiellement en Russie ; ils sont cinq fois plus nombreux si on compte les illégaux. 
Le troisième chapitre, radioscopie de la société russe, insiste sur sa profonde fragmentation. Les inégalités sociales, considérables, sont en constante augmentation depuis le marasme économique des années 1990. Elles apparaissent d'autant plus criantes à la génération qui a grandi avec l'Union soviétique, dont les institutions offrait une sécurité et un niveau de vie et de confort minimal à la très grande majorité des citoyens, expliquant ainsi certaines attitudes nostalgiques. Car si l'attention s'est majoritairement portée en occident sur la naissance d'un petit groupe d'ultra-riches, une couche significative d'extrêmepauvres s'est constituée au cours des vingt dernières années. L'éclosion d'une classe moyenne, maintes fois annoncée, est laborieuse. Celle-ci ne se repère pas tant par ses revenus que son mode de vie et représenterait 25 à $40 \%$ de la population totale. Mais le pays manque d'une force de travail qualifiée composée de professions intermédiaires et de cadres du secteur privé dont l'économie a cruellement besoin. Enfin la fracture entre urbains et ruraux semblent consommée. Si le dynamisme provincial est « essentiel à prendre en compte pour qui veut comprendre la Russie profonde qui se distingue nettement des capitales » (p. 66), la classe moyenne moscovite et pétersbourgeoise se détache et s'éloigne de plus en plus des réalités «d'une autre Russie $»^{3}$, celle des régions intermédiaires, et plus encore des régions dépressives du Nord et de Sibérie. Plus que jamais, un examen approfondi laisse place à « des Russies $»^{4}$ que tout oppose et explique la naissance d'un sentiment de rejet à l'égard de Moscou et des moscovites. L'écart se creuse également sur le plan des valeurs culturelles, les classes moyennes étant davantage pénétrées par les modèles étrangers (gastronomie, loisirs culturels, programmes télévisuels, etc.) que le reste de la population parmi laquelle les résistances russes sont plus ancrées.

L'analyse du régime politique (chapitre 4) ne fait évidemment pas l'économie de la genèse de l'image catastrophique dont joui la démocratie depuis les années 1990, en raison de son «[association] aux ravages du capitalisme » (p. 81). De la même manière, le totem mémoriel que constitue le coup de force de $1993^{5}$ annonce la présidentialisation quasi exponentielle du régime. De la fronde des régions de 1993-1994 à la crise économique

\footnotetext{
${ }^{3}$ En référence à l'ouvrage précédent de Jean Radvanyi : Retour d'une autre Russie. Une plongée dans le pays de Poutine, Lormont, Le Bord de l'eau, coll. « Clair \& net », 2013.

${ }^{4}$ Pour davantage de développements sur ce point, voir notamment : Raviot Jean-Robert, « Géographie politique de la Russie de $2010 »$, Hérodote 3/2010 (n 138) , p. 161-180.
} 
d'août 1998 ou encore le déclenchement de la 2nde guerre de Tchétchénie à l'été 1999, les auteurs mettent en perspective les principaux héritages et déterminants de la réalité politique du régime russe contemporain, qu'il s'agisse du centralisme autoritaire ou de la nécessité d'une «voie spécifique » pour la Russie. Les développements suivants s'attardent sur l'évolution du pouvoir poutinien, de la victoire du «centrisme patriotique » (p. 87) et des vertus stabilisatrices de la re-centralisation étatique sous l'effet de la « dictature de la loi » et de la «verticale du pouvoir» aux remous provoqués par les révolutions de couleur à ses frontières et le tournant conservateur à partir de 2012. Cette présentation se conclut par une réflexion sur l'avenir du régime russe et de son soutien populaire. Les auteurs se montrent en effet peu dupes de la « lune de miel» (p. 101) qui a suivi l'annexion de la Crimée et dont ils prédisent la fin imminente, précipitée notamment par l'absence de perspectives économiques. De la situation économique, il est justement question dans le chapitre suivant.

Cette cinquième section s'ouvre sur un extrait savoureux de la thèse de 3ème cycle de Vladimir Poutine au sujet de l'importance primordiale des matières premières et du rôle de l'État dans l'économie (p. 103). Les auteurs mettent en garde contre la tentation d'analyser la Russie à l'aune des modèles occidentaux. Longtemps comparé à la « maladie hollandaise », le «syndrome russe » est un mal bien particulier qui dépasse les seuls effets négatifs d'une économie de la rente au sein de laquelle les réformes de structure indispensables n'ont jamais été entreprises. Certes, les défis principaux de l'économie russe aujourd'hui sont la diversification de sa production industrielle et la hausse de sa productivité qui permettraient de la préserver davantage des effets des variations des cours des hydrocarbures. Néanmoins, le maintien des blocages structurels datant de l'époque de l'Union soviétique comme les déséquilibres persistants du commerce extérieur, ou le «serpent de mer du climat des affaires » (p. 136) et de la lutte contre la corruption apparaissent comme des chantiers colossaux, hors de portée du pouvoir politique. Le redressement, indéniable depuis les années 1990, s'est surtout fait par la reprise en main par l'État des principaux leviers de l'économie. Mais ce renforcement brutal du contrôle étatique et la création d'un capitalisme rentier aux mains d'un cercle de proches du Président de la fédération expliquent aussi les anomalies récurrentes de l'économie et le coût des infrastructures. Au total, si la Russie s'est

${ }^{5}$ Voir « Retour sur « Un octobre oublié ? La Russie de 1993 » avec Amandine Regamey et Carole Sigman » sur le carnet en ligne du labex TEPSIS pour une analyse détaillée (https://tepsis.hypotheses.org/892). 
hissée au 6ème rang des puissances économiques mondiales, elle est entrée en récession en 2015 et son PIB rapporté au nombre d'habitants la fait reculer de plus de 40 places.

Les deux derniers chapitres de l'ouvrage sont consacrés aux questions géopolitiques et de diplomatie. Ici encore les développements des auteurs laissent une large place à la synthèse des choix et des mutations des années 1990 et 2000. De l'échec de la Communauté des États indépendants (CEI) aux révolutions de couleur et l'exacerbation des tensions, les auteurs font le point sur le revirement diplomatique consécutif à l'épisode des sanctions et contres sanctions de 2014-2015. Décrypter le poutinisme sur la scène internationale et faire la part des choses entre la construction doctrinaire du nouveau régime et les bricolages idéologiques dépourvus de vision stratégique de long terme demeure une chose complexe. Une nouvelle vision du monde s'est toutefois fait jour et est désormais solidement structurée en Russie. Résolument offensive, cette puissance ré-émergente n'en est pas pour autant à court de solutions ou d'instruments. Sa stratégie de reconquête mondiale basée sur de nouveaux médias à vocation internationale et la mobilisation de sa diaspora ainsi que son alliance stratégique avec la Chine et les autres BRICS prouve qu'elle sait s'adapter et tirer parti de la nouvelle géométrie mondiale autant que des formes de guerre hybride dans le «nouveau grand jeu ». 